\title{
Refractory Leiomyosarcoma
}

National Cancer Institute

\section{Source}

National Cancer Institute. Refractory Leiomyosarcoma. NCI Thesaurus. Code C148294.

Leiomyosarcoma that does not respond to treatment. 